\title{
Técnica del tubo gástrico invertido como opción para la reconstrucción esofágica, en un hospital de tercer nivel en Bogotá, Colombia: presentación de dos casos y revisión de la literatura
}

\section{Two Cases of Reversed Gastric Tube (RGT) Esophagoplasty at a Third Level Hospital in Bogota, Colombia and Literature Review}

\author{
Carlos Leal Buitrago, MD, ${ }^{1}$ Felipe Bernal Santos, MD, ${ }^{2}$ Luis Felipe Cabrera Vargas, MD. ${ }^{3}$
}

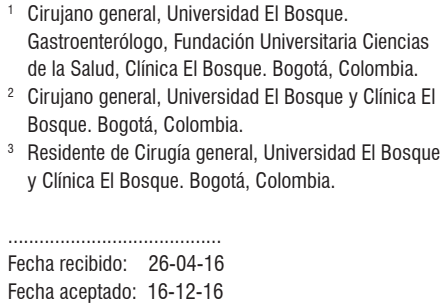

\begin{abstract}
Resumen
Introducción: las principales lesiones a nivel de la hipofaringe y el esófago son debidas a quemaduras por cáusticos y al cáncer esofágico. Estas pueden presentarse y comprometer las dos estructuras de un $17 \%$ a un $23 \%$, lo que genera la necesidad de una gran resección y, a su vez, el reto de una gran reconstrucción. En este estudio presentamos nuestra serie de casos empleando la técnica del tubo gástrico invertido y una revisión de la literatura con discusión crítica de los aspectos principales de este procedimiento. Métodos: pacientes en quienes se llevó a cabo reconstrucción esofágica mediante la técnica del tubo gástrico invertido, desde enero de 2010 hasta enero de 2015. Resultados: un paciente presentó estenosis de la anastomosis cervical, la cual fue manejada con dilataciones endoscópicas. Ninguno de los pacientes tuvo disfagia con las modificaciones en la dieta o síntomas clínicos de síndrome de Dumping o retardo en el vaciamiento gástrico. Sin embargo, los dos pacientes presentaron reflujo gástrico y requirieron un inhibidor de la bomba de protones (IBP). Discusión: el tubo gástrico invertido no se utiliza a menudo para la reconstrucción después de una esofagectomía total. Su ventaja sobre la técnica convencional de ascenso gástrico, la interposición de colon o el tubo gástrico invertido supercargado es que es una operación de una etapa y un procedimiento simple que requiere solamente una anastomosis. Puede ser transferido a la región cervical o incluso al esófago faríngeo para crear una anastomosis. Conclusiones: esta técnica permite la creación de un conducto más largo para la reconstrucción esofágica, con una tasa de complicaciones baja y ausencia de mortalidad.
\end{abstract}

Palabras clave

Tubo gástrico invertido, quemadura esofágica cáustica, cáncer esofágico.

\begin{abstract}
Introduction: The principal lesions in the hypharynx and esophagus are due to caustic burns and esophageal cancer which account for $17 \%$ to $23 \%$ of all events that compromise these two structures. They account for much of the surgery, especially for the challenge of major reconstruction. This study presents our series of cases using reversed gastric tube (RGT) esophagoplasty and presents a review of the literature and a critical discussion of the main aspects of this procedure. Methods: Patients underwent RGT esophagoplasties from January 2010 to January 2015. Results: One patient developed stenosis of the cervical anastomosis which was managed with endoscopic dilations. None of the patients developed dysphagia, clinical symptoms of dumping syndrome or delayed gastric emptying as the result of dietary modifications. Gastric reflux occurred in both patients and was teated with proton pump inhibitors. Discussion: RGT esophagoplasty is not often used for reconstruction after a total esophagectomy. Compared to the conventional technique of gastric ascent, interposition of the colon and supercharged reversed gastric tube techniques, it has the advantages that it is a one-step operation and is a simple procedure requiring only one anastomosis. It can be moved to the cervical region or even to the pharyngeal esophagus to create an anastomosis. Conclusions: This technique allows the creation of a longer duct for esophageal reconstruction and has a low complication rate with no mortality.
\end{abstract}

\section{Keywords}

Reversed gastric tube, caustic esophageal burn, esophageal cancer. 


\section{INTRODUCCIÓN}

Las principales lesiones a nivel de la hipofaringe y el esófago son debidas a quemaduras por cáusticos y al cáncer de esófago. Estas pueden presentarse y comprometer las dos estructuras de un $17 \%$ a un $23 \%$, lo que genera la necesidad de una gran resección y, a su vez, el reto de una gran reconstrucción, que consta de un remplazo exitoso del esófago y la restauración de la función faríngea. En estos casos se requiere un injerto de gran longitud para poder llevar a cabo la reconstrucción a nivel cervicofaríngeo, que aumenta el riesgo de isquemia distal. Inicialmente la interposición del colon era el método de elección para la reconstrucción de los defectos masivos como este; sin embargo, en algunos casos es necesaria la microcirugía. Presenta una alta incidencia de necrosis de aproximadamente un 5,8\% y las complicaciones son de difícil manejo $(1,2)$.

Por el contrario, el uso del estómago como injerto excluye la disección mesentérica, evita la anastomosis coloileal y la anastomosis cologástrica. Sin embargo, hay una limitación de longitud en el tubo gástrico no invertido y además está asociada con una gran morbilidad, con una incidencia global de complicaciones de un $26 \%$ a un $55 \%$, de las que la mediastinitis secundaria a la necrosis del injerto es la más catastrófica. La técnica del tubo gástrico invertido como opción en el remplazo del esófago, que emplea un tubo creado a partir de la curvatura mayor invertida, fue descrita por Beck y Carrell en 1905. No obstante, el primer caso exitoso de reconstrucción esofágica usando esta técnica en un humano fue reportado en 1951, por Gavriliu y Georgescu (3), quienes implementaron esta técnica en el 93,5\% de 768 pacientes de todas las edades, con patología esofágica benigna y maligna. Heimlich y Winfield $(4,5)$, en 1955, aplicaron este procedimiento en 8 perros, y en 1957 corroboraron la utilidad de esta técnica en la reconstrucción esofágica en humanos. En 1972 publicaron una serie de casos de 53 pacientes, lo que popularizó la técnica ya que presentaba ventajas tales como la posibilidad de alcanzar la región cervical y faríngea por cualquiera de las rutas mediastinales, anastomosis única y la restauración satisfactoria de la deglución. Sin embargo, la larga línea de suturas gástrica y el suministro sanguíneo irregular pueden considerarse como posibles desventajas. La presencia de fístula posoperatoria y de estenosis a nivel de la anastomosis se evidenció en un 7,5\% y $12,5 \%$ en la serie de Gavriliu y Georgescu (3). Se requieren más estudios para definir la aplicación del tubo gástrico invertido y mejorar el suministro sanguíneo para el segmento esofágico reconstruido. En este estudio presentamos dos casos en los que esta técnica fue empleada y una revisión de la literatura con discusión crítica de los aspectos principales de este procedimiento (5).

\section{PACIENTES Y MÉTODOS}

Pacientes en quienes se llevó a cabo la reconstrucción esofágica mediante la técnica del tubo gástrico invertido, desde enero de 2010 hasta enero de 2015. Dos pacientes fueron seleccionados (edades de 22 años y 46 años; hombres: 1; mujer: 1). Los dos casos fueron por quemadura hipofaríngea y esofágica por cáusticos. Se practicó esofagectomía total transhiatal con anastomosis cervical esofagogástrica a $3 \mathrm{~cm}$ del cricofaríngeo para la paciente femenina y anastomosis faringogástrica para el paciente masculino, con reconstrucción con tubo gástrico invertido, a causa de la resección masiva y la anastomosis alta. Todo lo anterior con previa firma del consentimiento informado y repleción nutricional con gastrostomía laparoscópica ubicada hacia la curvatura menor en el cuerpo gástrico. Las características clínicas de los pacientes se describen en la tabla 1.

\section{EVALUACIÓN PREOPERATORIA}

La evaluación y el manejo prequirúrgico de nuestros pacientes con quemadura esofágica por cáusticos, secundaria a intento de suicidio, fueron realizados inicialmente con una endoscopia de vías digestivas altas en las primeras 48 horas, para establecer el grado de severidad de la quemadura y así definir el pronóstico y la conducta a seguir. Después se inició el manejo médico con inhibidor de la bomba de protones (IBP) y antibioticoterapia, como lo recomiendan Conitini y colaboradores (6). No hubo administración de corticoides, ya que no han demostrado disminución del riesgo de estenosis a corto y mediano plazo, como lo describen Peclova y Fulton en dos revisiones sistemáticas $(7,8)$. Durante las primeras 72 horas los pacientes fueron mantenidos en vigilancia clínica continua en la unidad de cuidados intensivos intermedios. Se inició soporte metabólico con nutrición parenteral total y se realizó una endoscopia de vías digestivas altas de control entre la segunda y tercera semana del cuadro clínico para determinar la presencia de estenosis. Debido a la presencia de estenosis severa en nuestra serie de casos, hacia el día 21 se llevó a cabo una gastrostomía laparoscópica tipo Stamm, hacia la curvatura menor a nivel del cuerpo gástrico. Previo a la introducción de la sonda de nutrición, se practicó una endoscopia de vías digestivas altas intraoperatoriamente a través de la gastrotomía, con el fin de establecer la presencia de quemadura gástrica y la longitud de la quemadura y estenosis esofágica. Los pacientes fueron considerados como candidatos quirúrgicos, ya que no presentaban ninguna comorbilidad importante. Fueron valorados por el servicio de psiquiatría, que consideró que su estado mental era apto para manejar un postoperatorio de cirugía mayor y que deseaban restaurar su ingesta oral normal. 
Tabla 1. Características clínicas de los pacientes intervenidos con la técnica del tubo gástrico invertido

\begin{tabular}{|c|c|c|c|c|c|c|c|c|c|}
\hline n. ${ }^{\circ}$ & $\begin{array}{l}\text { Edad } \\
\text { (años) }\end{array}$ & Sexo & Causa & Severidad & $\begin{array}{c}\text { Grado de } \\
\text { estenosis a la } \\
\text { 3. }{ }^{\text {a }} \text { semana }\end{array}$ & $\begin{array}{l}\text { Longitud de la } \\
\text { quemadura }\end{array}$ & $\begin{array}{l}\text { Vía de nutrición } \\
\text { prequirúrgica }\end{array}$ & $\begin{array}{l}\text { Tiempo de } \\
\text { reconstrucción }\end{array}$ & $\begin{array}{c}\text { Vía de } \\
\text { reconstrucción }\end{array}$ \\
\hline 1 & 46 & M & $\begin{array}{l}\text { Quemadura } \\
\text { por cáusticos }\end{array}$ & IIB & $90 \%$ & $23 \mathrm{~cm}$ & $\begin{array}{l}\text { Gastrostomía } \\
\text { laparoscópica }\end{array}$ & 8 semanas & $\begin{array}{l}\text { Mediastino } \\
\text { posterior }\end{array}$ \\
\hline 2 & 22 & $\mathrm{~F}$ & $\begin{array}{l}\text { Quemadura } \\
\text { por cáusticos }\end{array}$ & IIIA & $95 \%$ & $27 \mathrm{~cm}$ & $\begin{array}{l}\text { Gastrostomía } \\
\text { laparoscópica }\end{array}$ & 8 semanas & $\begin{array}{l}\text { Mediastino } \\
\text { posterior }\end{array}$ \\
\hline
\end{tabular}

\section{TÉCNICA QUIRÚRGICA}

Los pacientes fueron colocados en decúbito supino. Inicialmente se llevó acabo la etapa abdominal mediante laparotomía mediana, con disección por planos hasta la cavidad abdominal. Se retira la sonda de gastrostomía y se cierra la fístula gástrica con sutura de polidioxanona 3-0 (Ethicon, Inc., Somerville, NJ, USA). Se disecciona la arteria gastroepiploica izquierda lo más cercano a su origen en la arteria esplénica, conservando su irrigación a la curvatura mayor del estómago, y se ligan y cortan los vasos cortos con bisturí armónico (Ethicon, Inc., Somerville, NJ, USA), para dar más movilidad al fondo gástrico. Se identifica la unión entre la arteria gastroepiploica izquierda y la derecha y en este punto, en la curvatura mayor gástrica, se realizan dos disparos con sutura mecánica lineal cortante de $55 \mathrm{~mm}$ (Ethicon, Inc., Somerville, NJ, USA), con dirección cefálica hacia el punto medio del fondo gástrico, sin seccionarlo, con un margen de $2 \mathrm{~cm}$ aproximadamente, con liberación de las adherencias fúndicas gástricas al diafragma, lo que crea el tubo gástrico invertido (figuras 1, 2 y 3); no se hizo refuerzo manual de la línea de sutura mecánica gástrica. Además se llevó a cabo una maniobra de Kocher extensa para liberar el duodeno y la cabeza del páncreas, con el fin de aproximar el píloro hacia la línea media. Una vez hecho esto, se pasó a la etapa cervical, en la que mediante cervicotomía izquierda, disección por planos hasta el espacio paravertebral izquierdo e identificación del esófago en su porción proximal se realizó una sección de este con bisturí frio, superior al punto de la estenosis por cáusticos, aproximadamente entre $2 \mathrm{a} 4 \mathrm{~cm}$ después de cricofaríngeo. Por vía transhiatal se llevó a cabo la extracción del esófago más un disparo con sutura mecánica lineal cortante de $55 \mathrm{~mm}$ (Ethicon, Inc., Somerville, NJ, USA) a nivel de la unión esofagogástrica, para completar la esofagectomía total. Luego se procedió a una piloroplastia tipo Heineke Mikulicz para evitar un síndrome de retardo en el vaciamiento gástrico debido a la sección troncular del nervio vago (figura 4). El tubo gástrico invertido fue pediculado con la arteria gastroepiploica izquierda a través del mediastino posterior, para prevenir cualquier tipo de torsión y evitar la tensión desme- dida en el pedículo vascular distal, mediante dedo disección hasta alcanzar la región cervical (figura 5). Allí se llevó a cabo una esofagogastroanastomosis terminoterminal manual en dos planos (mucomucoso y seromuscular) con sutura polidioxanona 3-0 (Ethicon, Inc., Somerville, NJ, USA). Bajo visión y control directo, se avanzó una sonda nasogástrica para descompresión y una sonda nasoyeyunal avanzada para nutrición enteral temprana (figuras 6 y 7). Se dejó un dren de Penrose como testigo a nivel del sitio de la anastomosis (figura 8). La nutrición enteral avanzada se inició al tercer día postoperatorio. Todos los pacientes requirieron monitorización postoperatoria en unidad de cuidados intensivos (UCI). Al séptimo día postoperatorio, se les realizó una radiografía de vías digestivas altas de rutina para establecer o no la presencia de fugas y poder iniciar la vía oral. Se hizo una endoscopia de vías digestivas altas de control a las 2 semanas después de la cirugía para inspeccionar la anastomosis y la línea de suturas del estómago invertido con el fin de descartar fístulas o mucosa mal perfundida.

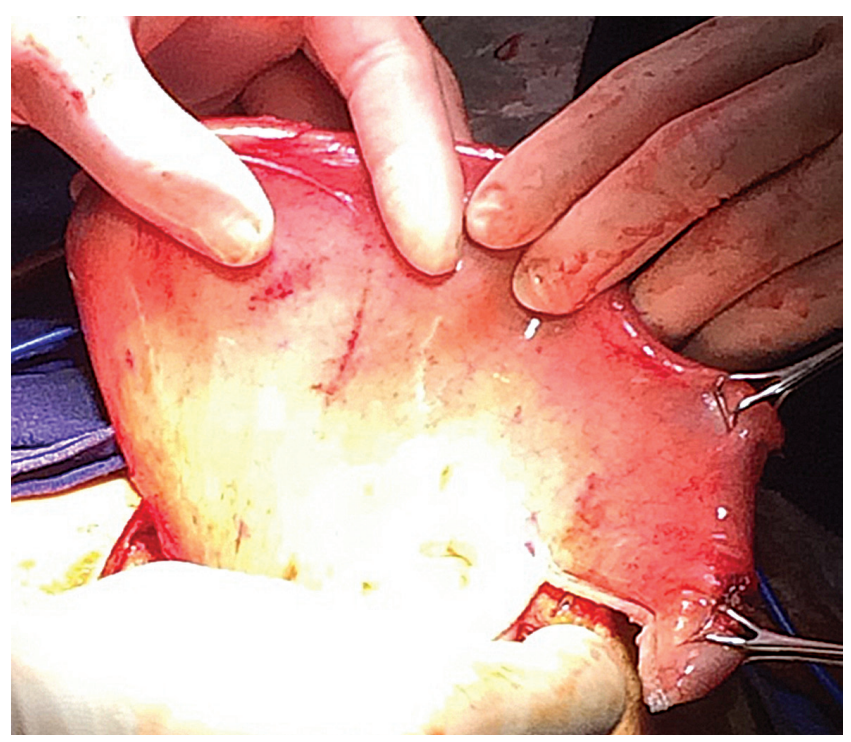

Figura 1. Identificación de la unión entre la arteria gastroepiploica izquierda y la derecha para realizar el primer disparo de sutura mecánica lineal cortante. 


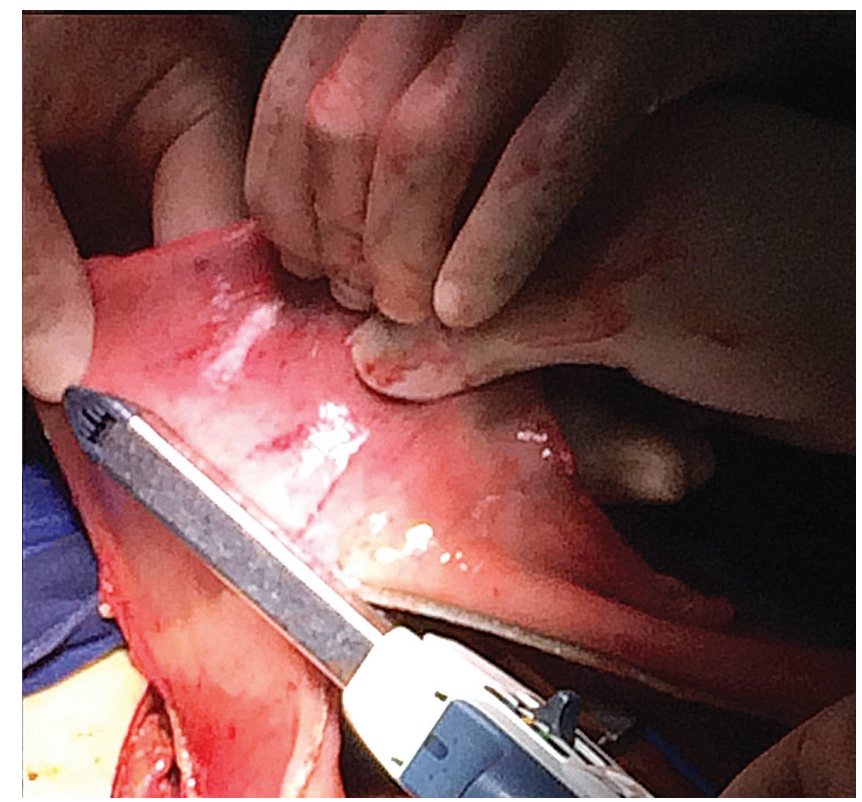

Figura 2. Creación del tubo gástrico invertido con sutura mecánica lineal cortante.
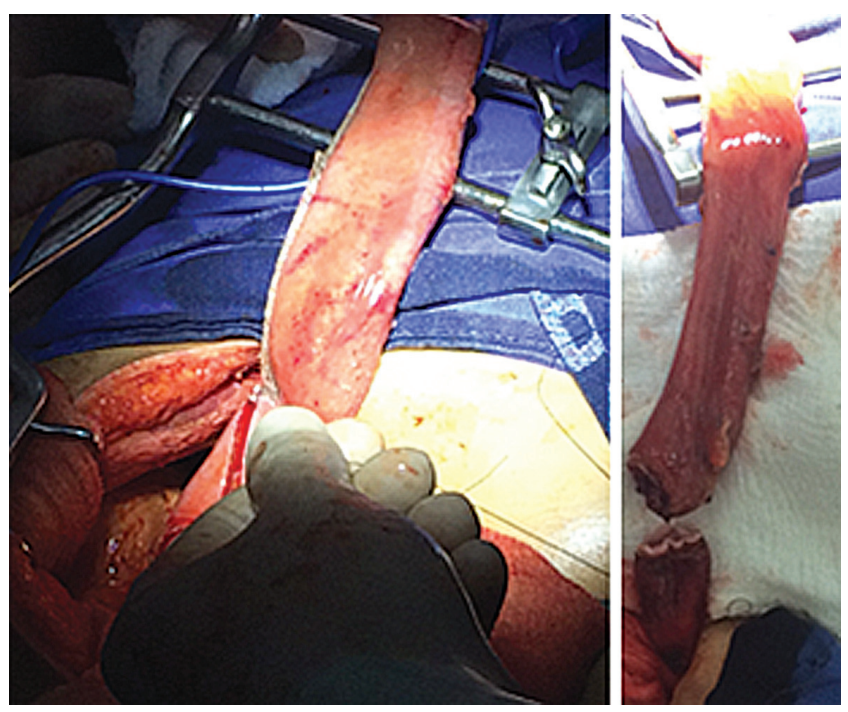

Figura 3. Tubo gástrico invertido que alcanza con facilidad la región cervical por vía subcutánea.

\section{RESULTADOS}

La longitud del injerto de tubo gástrico invertido fue medida por vía endoscópica a las 2 semanas del postoperatorio, con un promedio de $32,5 \mathrm{~cm}$. La estadía en la UCI varió de 4 a 5 días sin ninguna mortalidad. Los dos procedimientos se pudieron llevar acabo sin ninguna complicación intraoperatoria. La fístula postoperatoria de la línea de sutura mecánica en el estómago invertido presentada en la paciente femenina a $4 \mathrm{~cm}$ de la esofagogastroanastomosis resolvió con manejo médico dado por nutrición parenteral total, antibioticoterapia de amplio espectro y toracostomía cerrada para drenaje del espacio pleural derecho, sin otras repercusiones clínicas después de 2 semanas. Es de resaltar que ninguno de nuestros pacientes tuvo neumotórax perioperatorio. El paciente masculino presentó estenosis a nivel de la faringogastroanastomosis cervical de forma tardía, la cual resolvió completamente con dilataciones neumáticas endoscópicas sin disfagia a largo plazo. El dren de Penrose se retiró al séptimo día y se inició dieta corriente hacia el décimo día con ulterior retiro de las sondas para nutrición sin ninguna complicación. Ninguno de los pacientes mostró disfagia con las modificaciones en la dieta o síntomas clínicos de síndrome de Dumping o retardo en el vaciamiento gástrico. Sin embargo, hubo reflujo gástrico en los dos pacientes, que requirió IBP permanente con elevación de la cabecera A 30 grados (tabla 2).

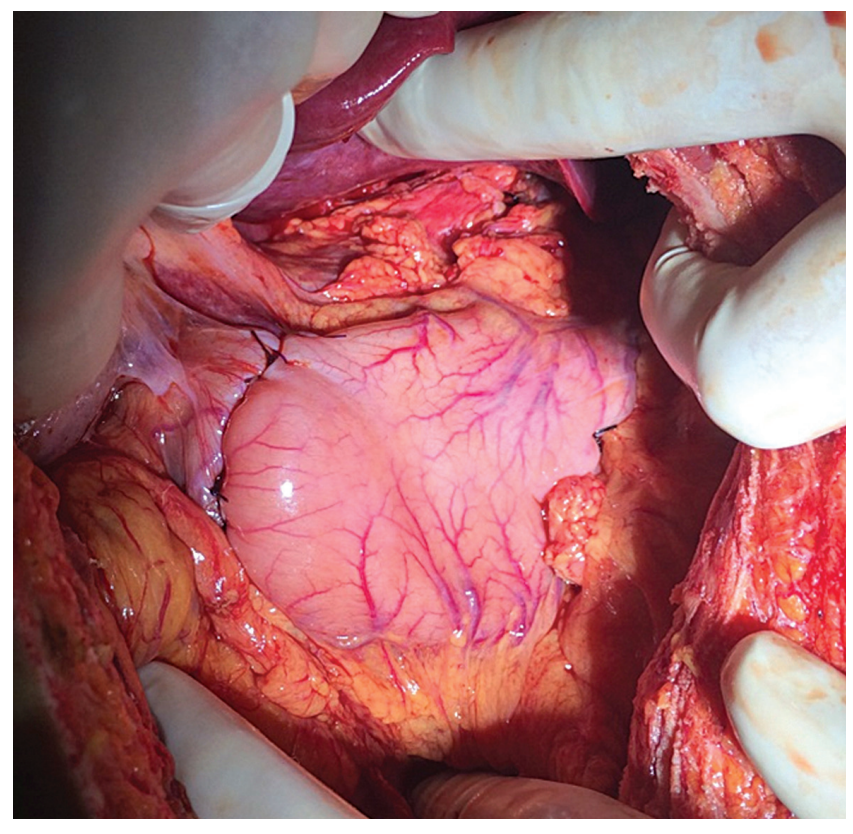

Figura 4. Piloroplastia para evitar el síndrome de retardo en el vaciamiento gástrico.

\section{DISCUSIÓN}

Las quemaduras esofágicas por cáusticos ocurren por ingesta accidental en un $80 \%$ de la población pediátrica; sin embargo, el $20 \%$ restante se presenta como intento de suicidio en la población adulta, como se observa en nuestros dos casos. La reconstrucción ulterior a una esofagectomía total se realizaba de forma rutinaria con la interposición de colon, ya que este es lo suficientemente largo como para reemplazar el segmento resecado; no obstante, la complicada manipulación quirúrgica era una desventaja para esta 


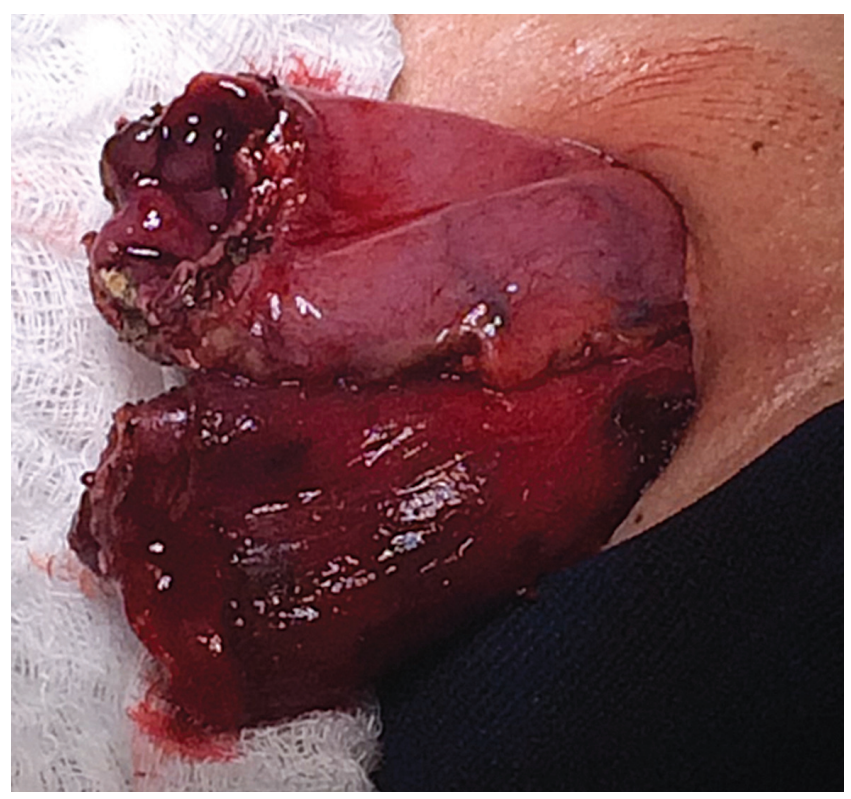

Figura 5. Tubo gástrico invertido pediculado con la arteria gastroepiploica izquierda a través del mediastino posterior hasta la región cervical.

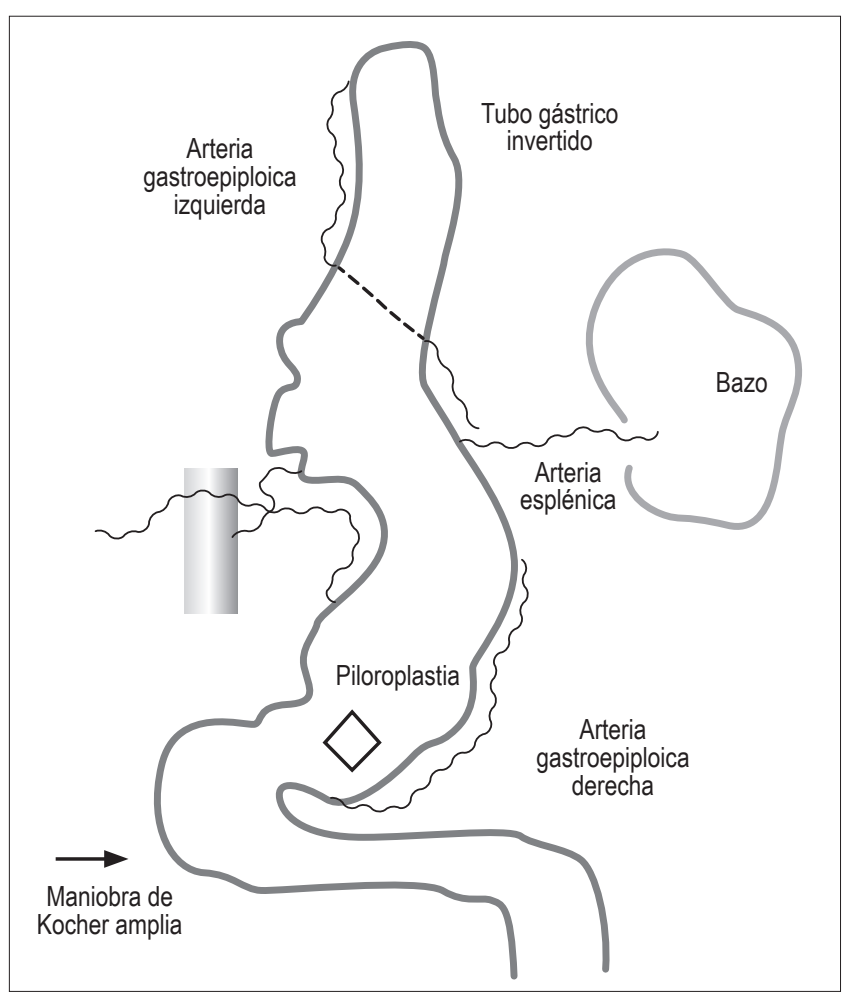

Figura 7. Tubo gástrico invertido conformado.

técnica, como lo reportó Knezevic (9) y colaboradores, con una incidencia de complicaciones tempranas (filtración de

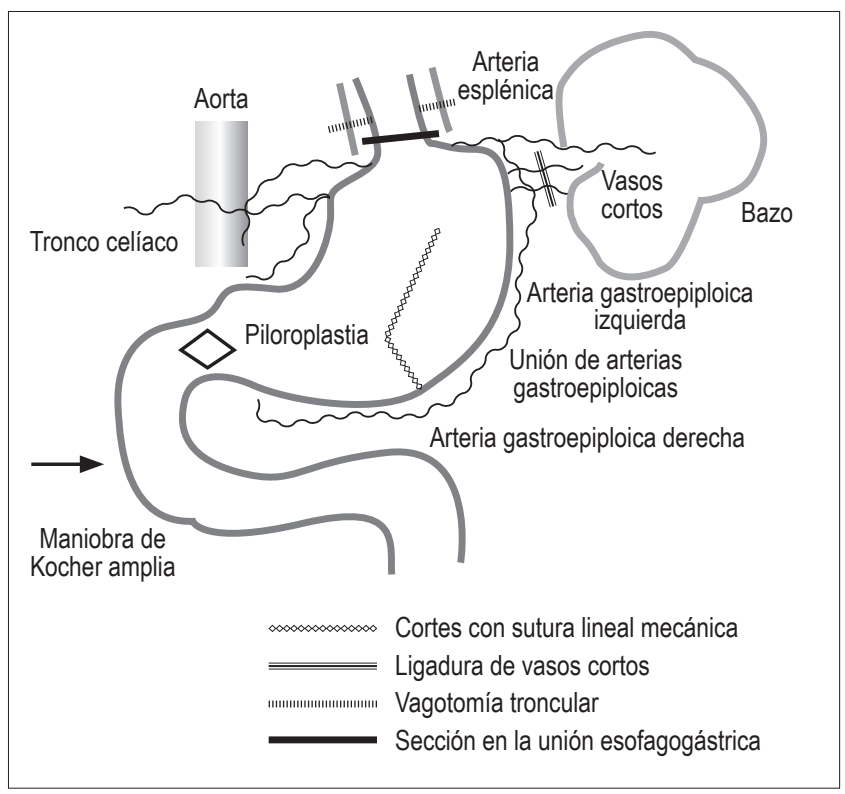

Figura 6. Esquema quirúrgico para la creación del tubo gástrico invertido.

la anastomosis, necrosis colónica y hemoneumotórax), hasta de un 26,4\%, y complicaciones tardías (estenosis de la anastomosis, úlcera péptica del colon, obstrucción intestinal y compresión de la salida torácica), en un $14 \%$. Por lo anterior, el estómago es utilizado ampliamente como la primera opción para corregir este tipo de defectos anatómicos después de una resección esofágica, y el colon se usa solo en pacientes previamente gastrectomizados, aquellos que requieren gastrectomía simultánea a la esofagectomía y los que presentan quemaduras extensas de la mucosa gástrica por cáusticos. Por otro lado, las dificultades aumentan con la altura de la anastomosis, con filtraciones y estenosis más frecuentes. Para minimizar los problemas de dehiscencia anastomótica y necrosis del injerto debido al aumento de la tensión y a la mala irrigación sanguínea, se han diseñado otras técnicas para incrementar la vascularización de la región reconstruida, como lo son el tubo gástrico invertido o el tubo gástrico invertido supercargado, el cual emplea anastomosis microvasculares a nivel cervical que usan la arteria gástrica izquierda y procedimientos de acondicionamiento gástrico como el descrito por Akiyama (10) y colaboradores, basados en el fenómeno de retraso. Estos llevaron a cabo una embolización percutánea de los vasos gástricos, con el fin de generar circulación colateral por difusión gástrica sanguínea y de esta forma mejorar la irrigación del tubo gástrico a ascender (11).

El tubo gástrico invertido no se utiliza a menudo para la reconstrucción después de una esofagectomía total; sin embargo, puede presentar algunas ventajas sobre la 

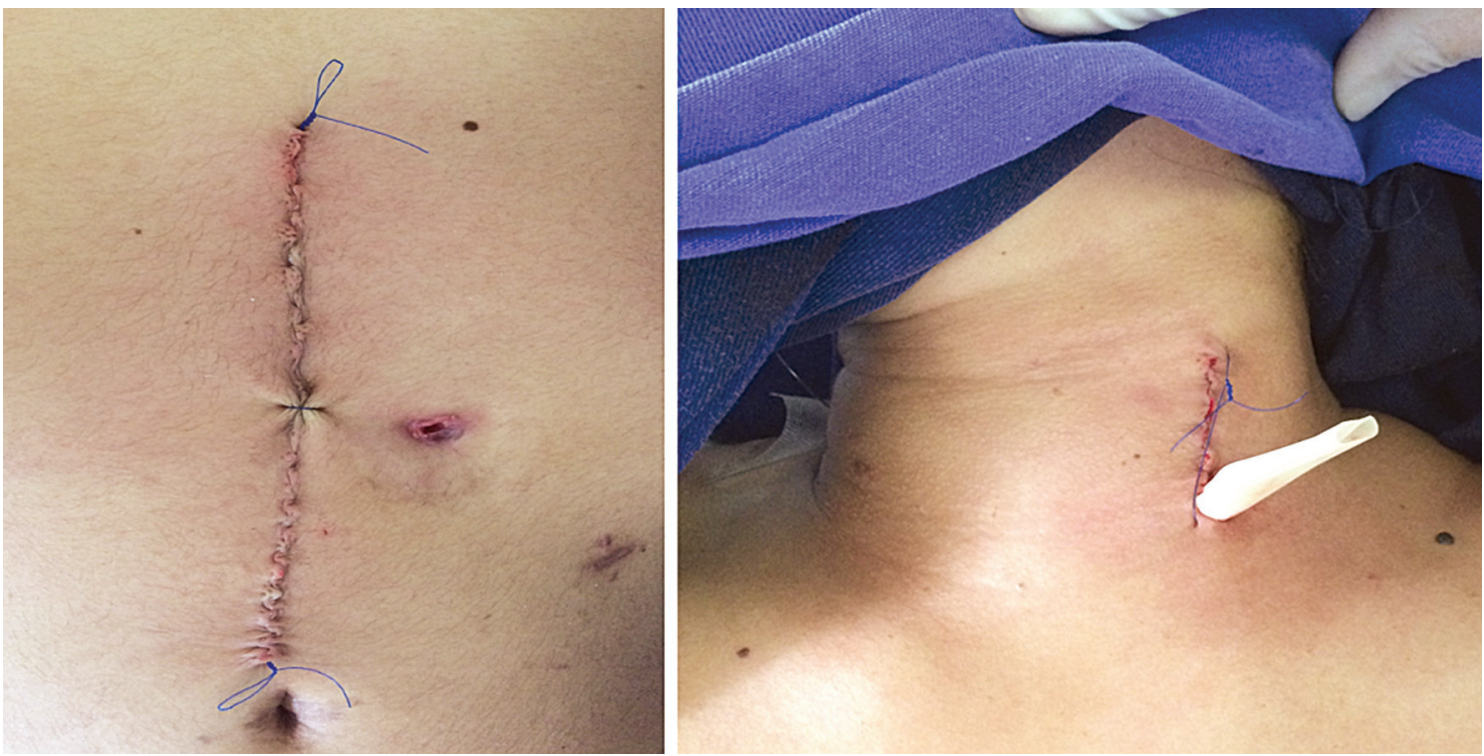

Figura 8. Heridas quirúrgicas.

Tabla 2. Resultados

\begin{tabular}{|c|c|c|c|c|c|c|c|c|c|c|c|}
\hline $\mathrm{n} .^{\circ}$ & $\begin{array}{l}\text { Longitud } \\
\text { del } \\
\text { injerto } \\
\text { (cm) }\end{array}$ & $\begin{array}{c}\text { Tiempo } \\
\text { quirúrgico }\end{array}$ & $\begin{array}{c}\text { Tipo de } \\
\text { anastomosis }\end{array}$ & Sangrado & $\begin{array}{c}\text { Prueba } \\
\text { anastomótica }\end{array}$ & $\begin{array}{c}\text { Estancia } \\
\text { en UCl }\end{array}$ & Complicaciones & Manejo & $\begin{array}{c}\text { Estancia } \\
\text { hospita- } \\
\text { laria }\end{array}$ & $\begin{array}{l}\text { Segui- } \\
\text { miento }\end{array}$ & Evolución \\
\hline 1 & 35 & $\begin{array}{c}2 \mathrm{~h} \text { y } 45 \\
\min \end{array}$ & $\begin{array}{l}\text { Terminoter- } \\
\text { minal cervical } \\
\text { en un plano } \\
\text { faringogás- } \\
\text { trica }\end{array}$ & $250 \mathrm{~mL}$ & $\begin{array}{l}\text { Esofagograma } \\
\text { al } 7 .^{\circ} \text { día: } \\
\text { negativo }\end{array}$ & 4 días & $\begin{array}{l}\text { Estenosis tardía } \\
(6 \mathrm{~m}) \text { de la } \\
\text { anastomosis } \\
\text { cervical }\end{array}$ & $\begin{array}{l}3 \text { sesiones de } \\
\text { dilataciones } \\
\text { neumáticas } \\
\text { endoscópicas }\end{array}$ & 15 días & 2 años & $\begin{array}{l}\text { Tolerando } \\
\text { dieta regu- } \\
\text { lar, reflujo } \\
\text { gástrico }\end{array}$ \\
\hline 2 & 32 & $\begin{array}{c}2 \text { h y } 30 \\
\text { min }\end{array}$ & $\begin{array}{l}\text { Terminoter- } \\
\text { minal cervical } \\
\text { en dos } \\
\text { planos a } 3 \\
\text { cm del crico- } \\
\text { faríngeo }\end{array}$ & $300 \mathrm{~mL}$ & $\begin{array}{l}\text { Esofagograma } \\
\text { al } 7 .^{\circ} \text { dia: } \\
\text { positivo }\end{array}$ & 5 días & $\begin{array}{l}\text { Filtración de la } \\
\text { línea de grapado } \\
\text { gástrico mínima } \\
\text { a } 4 \mathrm{~cm} \text { de la } \\
\text { anastomosis } \\
\text { cervical }\end{array}$ & $\begin{array}{l}\text { Antibioti- } \\
\text { coterapia, } \\
\text { nutrición } \\
\text { parenteral } \\
\text { total más } \\
\text { toracosto- } \\
\text { mía cerrada } \\
\text { derecha }\end{array}$ & 21 días & $\begin{array}{c}6 \\
\text { meses }\end{array}$ & $\begin{array}{l}\text { Tolerando } \\
\text { dieta regu- } \\
\text { lar, reflujo } \\
\text { gástrico }\end{array}$ \\
\hline
\end{tabular}

técnica convencional de ascenso gástrico, la interposición de colon o el tubo gástrico invertido supercargado, ya que es un procedimiento simple que requiere solamente una anastomosis a nivel cervical. Puede ser transferido a la región cervical o incluso al esófago faríngeo para crear una anastomosis. Debido a que el calibre del tubo gástrico invertido es variable, puede aproximarse más al del esófago proximal o incluso al de la orofaringe, lo que disminuye la posibilidad de estenosis postoperatoria de la anastomosis, la cual, en reconstrucción esofágica, está reportada por Honkoop (12) y colaboradores hasta en un $42 \%$. Además, el tubo gástrico invertido facilita un proceso de deglución más fisiológico y permite un mayor aclaramiento esofá- gico. El pedículo vascular del tubo gástrico invertido dado por la arteria gastroepiploica izquierda evita la necesidad de cirugía microvascular, disminuye el riesgo de necrosis y filtración de la anastomosis, que se encuentra descrito por Orringer (13) y colaboradores hasta en un 13\%; sin embargo, uno de nuestros pacientes presentó una filtración a nivel de la línea de sutura mecánica del tubo gástrico invertido proximal a la anastomosis, la cual resolvió con manejo médico con toracostomía a drenaje cerrado derecha y no requirió re intervención quirúrgica. Parte del epiplón mayor que se asciende con la curvatura mayor del tubo gástrico invertido ofrece una cobertura ideal del injerto y llena todos los espacios muertos en el mediastino posterior. 
El injerto puede ser ascendido gracias a su gran longitud a través del mediastino posterior, retroesternal o incluso de forma subcutánea, aunque esta última vía presenta un alto riesgo de obstrucción debido a la compresión de las prominencias óseas, como se empleó en nuestros pacientes. Es importante definir que el tiempo quirúrgico y el sangrado son similares al del ascenso gástrico convencional $(14,15)$.

Por otra parte, las desventajas del tubo gástrico invertido incluyen la secreción excesiva de ácido gástrico, aunque la anastomosis esofagogástrica del tubo gástrico invertido implica una porción del estómago con secreción baja de ácido, que favorece el reflujo gastrofaríngeo, que requiere la administración de un IBP de forma prolongada y la cabecera a 30 grados, como en nuestros pacientes $(16,17)$.

La mayoría de los estudios publicados que usaron esta técnica fue realizada en población pediátrica. El Dr. Ein (18) de Toronto publicó una serie de casos, recolectada en 32 años, de 11 pacientes que habían sido sometidos a reemplazo esofágico con tubos gástricos, de los cuales 8 eran antiperistálticos (invertidos) y 3 eran isoperistálticos. Inicialmente 6 pacientes requirieron dilataciones; sin embargo, el seguimiento a largo plazo a 30 años reveló que 9 de 11 pacientes estaban deglutiendo normalmente. En nuestro análisis de resultados, solo 1 paciente requirió dilatación neumática endoscópica y todos los pacientes están actualmente tolerando una dieta regular sin ningún síntoma de disfagia y con el reflujo gastrofaríngeo controlado con el IBP, sin necesidad de soporte nutricional parenteral o enteral. Es importante recalcar que la gran longitud del injerto gástrico obtenida con esta técnica permite emplear cualquiera de las vías torácicas (mediastino posterior, retroesternal y subcutánea); además es una técnica factible, la cual es reproducible por cualquier cirujano general ya que no requiere cirugía microvascular, como en la transposición de yeyuno, siempre teniendo precaución en mantener un diámetro aceptable $(3 \mathrm{~cm})$ al conformar el tubo gástrico invertido. El seguimiento a largo plazo requiere menos experticia, lo que facilita la realización de las endoscopias de vías digestivas altas en centros de mediana a baja complejidad (19-21).

\section{CONCLUSIONES}

Este procedimiento dio resultados favorables. A pesar de ser únicamente dos casos, podemos concluir que la técnica del tubo gástrico invertido permite la creación de un conducto más largo para el reemplazo de todo el esófago, con ausencia de mortalidad y un tiempo de hospitalización moderado. Aunque la experiencia en adultos es baja, es un procedimiento de reconstrucción seguro, reproducible y una prometedora opción para la cirugía de reconstrucción faringoesofágica.

\section{Conflictos de interés}

No declarados.

\section{REFERENCIAS}

1. Tiedeken J, Uradomo L, Anderson K. Minimally invasive repair of a late stricture in a reversed gastric tube. J Pediatr Surg. 2012;47:2321-6.

2. Dale WA, Sherman DC. Late reconstruction of congenital esophageal atresia by intrathoracic colon transplantation. J Thorac Surg. 1955;29:344-56.

3. Gavriliu D, Georgescue L. Esophagoplastic direction a material gastric. Rev Stiintelor Med (Bucharest). 1955;3:33.

4. Heimlich HJ. The use of a gastric tube to replace the esophagus as performed by Dr. Dan Gavriluiu of Bucharest, Romania. Surgery. 1957;42:693-5.

5. Heimlich HJ, Winfield JM. The use of a gastric tube to replace or bypass the esophagus. Surgery. 1955;37:549-59.

6. Contini S, Scarpignato C. Caustic injury of the upper gastrointestinal tract: A comprehensive review. World J Gastroenterol. 2013;19(25):3918-30.

7. Pelclová $\mathrm{D}$, Navrátil T. Do corticosteroids prevent oesophageal stricture after corrosive ingestion? Toxicol Rev. 2005;24(2):125-9.

8. Fulton JA, Hoffman RS. Steroids in second degree caustic burns of the esophagus: a systematic pooled analysis of fifty years of human data: 1956-2006. Clin Toxicol (Phila). 2007;45(4):402-8.

9. Knezevi JD, Radovanovi NS, Simi AP, et al. Colon interposition in the treatment of esophageal caustic strictures: 40 years of experience. Dis Esophagus. 2007;20(6):530-4.

10. Reavis K, Chang E, Jobe B, et al. Utilization of the delay phenomenon improves blood flow and reduces collagen deposition in esophagogastric anastomoses. Ann Surg. 2005;241(5):736-47.

11. Hölscher A, Schneider P, Gutschow C, et al. Laparoscopic ischemic conditioning of the stomach for esophageal replacement. Ann Surg. 2007;245(2):241-6.

12. Honkoop P, Siersema PD, Tilanus HW, et al. Benign anastomotic strictures after transhiatal esophagectomy and cervical esophagogastrostomy: risk factors and management. J Thorac Cardiovasc Surg. 1996;111(6):1141-6.

13. Orringer MB, Marshall B, Iannettoni MD. Transhiatal esophagectomy for treatment of benign and malignant esophageal disease. World J Surg. 2001;25(2):196-203.

14. Harlak A, Yigit T, Coskun K. Surgical treatment of caustic esophageal strictures in adults. Int J Surg. 2013;11(2):164-8.

15. Gupta L, Bhatnagar V, Gupta AK. Long-term follow-up of patients with esophageal replacement by reversed gastric tube. Eur J Pediatr Surg. 2011;21(2):88-93.

16. Huang P, Chen C, Yang T. Supercharged reversed gastric tube technique: a microvascular anastomosis procedure for pharyngo-oesophageal reconstruction after total laryngopharyngo-oesophagectomy. Eur J Cardiothorac Surg. $2013 ; 44(2): 258-62$. 
17. Murakami M, Sugiyama A, Ikegami T, etal. Revascularization using the short gastric vessels of the gastric tube after subtotal esophagectomy for intrathoracic esophageal carcinoma. J Am Coll Surg. 2000; 190:71-7.

18. Ein SH, Shandling B, Stephens CA. Twenty one year experience with the pediatric gastric tube. J Pediatr Surg. 1987;22:77-81.

19. Sitges AC, Sanchez-ortega JM, Sitges AS. Late complications of reversed gastric tube esophagoplasty. Thorax. 1986;41:61-5.
20. Guzmán-Chávez OR, Bautista-González S, Ramírez-Solís A. Manejo quirúrgico de reconstrucción esofágica en pacientes con estenosis esofágica por cáusticos. Rev Med MD 2012;3(4):211-6.

21. Mendez M, Perez A, Perez D. Quemadura esofágica por ingesta de cáusticos en una población pediátrica Hospital de la Misericordia - Bogotá 1996 al 2009. CIRUPED. 2012;2(2). 